\title{
Swine-Origin Influenza A (H1N1) and Seasonal Influenza in Qazvin Province, Iran: Comparison of Epidemiological Features, Clinical Manifestations and Outcome of the 2009 Pandemic
}

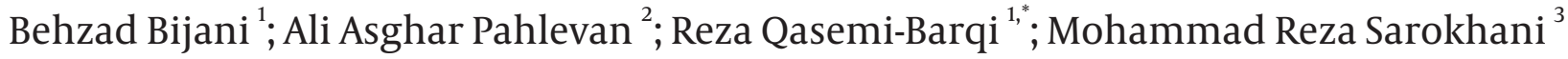 \\ ${ }_{1}^{1}$ Department of Infectious Diseases, Faculty of Medicine, Qazvin University of Medical Sciences, Qazvin, IR Iran \\ ${ }^{2}$ Department of Microbiology, Faculty of Medicine, Qazvin University of Medical Sciences, Qazvin, IR Iran \\ ${ }^{3}$ Department of Biotechnology, Faculty of Health and Paramedical Sciences, Qazvin University of Medical Sciences, Qazvin, IR Iran \\ ${ }^{*}$ Corresponding author: Reza Qasemi-Barqi, Department of Infectious Diseases, Faculty of Medicine, Qazvin University of Medical Sciences, Qazvin, IR Iran. Tel: +98-2833326032, Fax: \\ +98-2833326033, E-mail: rqasemi.barqi@yahoo.com
}

Received: December 19, 2014; Accepted: April 15, 2015

\begin{abstract}
Background: Emergence of a novel swine-origin influenza A (H1N1) pandemic since 2009 attracted the attention of scientists to characterize epidemiological features and clinical manifestations of this disease in comparison to seasonal flu in different parts of the world.

Objectives: The goal of this investigation was to compare these features in confirmed cases of swine-origin influenza A(H1N1) and seasonal flu in the 2009 epidemic in Qazvin province, Iran.

Patients and Methods: This cross sectional study was performed during 2009 in the Qazvin province. The epidemiological characteristics and clinical manifestations of all cases with severe flu-like manifestations were registered. Diagnosis of confirmed cases of both groups was performed by Real Time-Polymerase Chain Reaction (RT-PCR) on respiratory secretions of positive cases of swine-origin influenza A (H1N1) and seasonal influenza that were entered in the study. Analysis of quantitative data was performed using paired t-test and those of qualitative variables by chi square and Fisher's exact test.

Results: Among a total of 518 patients with clinical signs of severe influenza throughout the Qazvin province, 76 confirmed cases of swineorigin influenza A (H1N1) and 36 cases of other types of influenza A (seasonal influenza) were detected. The mean age of the first group was $25.67 \pm 16.9$ years and that of the second group was $36.03 \pm 19.8$, with a significant difference between the two groups $(\mathrm{P}<0.01)$. The appearance of diarrhea was significantly higher in patients with swine-origin influenza compared to those with seasonal influenza $(\mathrm{P}<$ 0.005). There was no statistically significant difference in the number of hospitalizations, need for intensive care, assisted ventilation, and mortality rate between the two groups.

Conclusions: Higher prevalence of disease in younger individuals, higher rate of gastrointestinal manifestations and occurrence outside of the epidemic season, were the most important characteristics of swine-origin influenza in comparison to seasonal influenza, in the 2009 pandemic of Qazvin province, Iran.
\end{abstract}

Keywords: Influenza A Virus, H1N1 Subtype; Epidemiology

\section{Background}

Epidemiological features and clinical symptoms of seasonal influenza have been thoroughly studied and well known for many years. Epidemics of influenza within the recent years have caused numerous absences from work and even death worldwide. The majority of severe cases of disease and mortalities occur in old people and the secondary bacterial pneumonia is regarded as the major cause of death (1). Within the past few years, repeated antigenic drifts have occurred in seasonal influenza viruses leading to outbreaks of disease every three to four years. However, no obvious change in epidemiological features and clinical manifestations of the disease was reported. During the spring of 2009, numerous cases of influenza outside the flu season and mostly with different epidemiological features, especially higher incidence and more severe cases amongst the younger age group were described. The etiological agent was a new influenza virus, which was named as the swine origin novel influenza A (H1N1) virus (SO-IV) because of its antigenic similarity to North American swine flu viruses. Following the identification of the genetic content of this virus, it became evident that the genomic content was different from all other viruses isolated from human and animals so far (2). Later, it became evident that the new virus had emerged through reassortment of two swine viruses, a human virus, and an avian virus yet with a genome mostly originated from the swine virus. The first confirmed case of the disease in the Qazvin province was reported during July 2009 followed by another 76 confirmed cases of disease throughout the province.

\section{Objectives}

Considering the international reaction towards the emergence of this pandemic and the epidemiological diversities between novel and seasonal influenza, and also extensive global concentration on symptoms of disease, the present study focused on comparing the epidemio-

Copyright (C) 2015, School of Paramedical Sciences, Qazvin University of Medical Sciences. This is an open-access article distributed under the terms of the Creative Commons Attribution-NonCommercial 4.0 International License (http://creativecommons.org/licenses/by-nc/4.0/) which permits copy and redistribute the material just in noncommercial usages, provided the original work is properly cited. 
logical features, clinical manifestations, and the final outcome of the swine-origin influenza A (H1N1) compared with seasonal influenza in the Qazvin province. Our research is the only study so far, comparing confirmed cases of seasonal influenza with swine-origin influenza A (H1N1) in the Eastern Mediterranean region.

\section{Patients and Methods}

\subsection{Study Design}

This was a descriptive cross-sectional study carried out between July and December 2009 in the province of Qazvin (Iran). Data were collected through the national influenza follow-up program carried out by the Ministry of Health and Medical Education in cooperation with the universities of medical sciences throughout the country (3). Sampling of respiratory secretions was performed for patients with clinical manifestations of severe influenza, who had visited the health centers within the province during this period. A confirmed case of influenza was defined as a patient with fever, moderate to severe respiratory symptoms, and positive RT-PCR test performed on respiratory secretions. Demographic characteristics, epidemiological features, clinical manifestations, and the course and outcome of the disease were recorded in patient surveillance forms by health officers and medical staff of the health centers and referral hospitals.

\subsection{Laboratory Analysis}

Samples were inoculated into Dulbecco's modified Eagle's medium and transferred to the molecular division of the province's reference laboratory and kept at $70^{\circ} \mathrm{C}$ until DNA extraction. Laboratory diagnosis of etiological agents was accomplished by the Real Time-Polymerase Chain Reaction (RT-PCR) technique using one and twostage commercial PCR kits (Qiagen, Germany and Invitrogen, Brazil) and application of three sets of primers; specific probes for universal influenza A virus, swine influenza A virus, and swine $\mathrm{H} 1 \mathrm{~N} 1$ virus, according to the World Health Organization (WHO) instructions (4). The specimens that produced a positive reaction with all three sets of primers were considered as having the swine-origin influenza A (H1N1) virus and those with no polymerization reaction with all three primers were regarded as negative. The virus strains that only produced a positive reaction with the universal influenza A primer were considered as the seasonal influenza virus.

\subsection{Statistical Analysis}

Data were analyzed by Epi info ${ }^{\mathrm{TM}}$ version 3.5.1 software using paired t-test, chi square and Fisher's exact test. P values less than 0.05 were considered statistically significant.

\section{Results}

Between July and December 2009, out of 518 tested pa- tients, 76 confirmed cases of swine-origin influenza A (H1N1) and 36 confirmed cases of infection with other types of influenza A viruses (seasonal flu) were detected. Ten hospitals in five cities within the province were in charge of providing inpatient facilities to the patients. The incidence of disease during different months is shown in Figure 1. The age range of patients with pandemic influenza was between two months and 72 years with a median age of 23 years and mean age of $25.67 \pm 16.9$ years, while in those with seasonal influenza the age range, median age, and mean age were one month to 80 years, 30 years, and $36.03 \pm 19.8$ years, respectively. The difference in mean age of patients with pandemic influenza and those with seasonal influenza was statistically significant $(\mathrm{P}=0.005)$. Table 1 presents information on the studied subjects, including age, gender, contact with similar patients, and traveling abroad. As shown by Table 1 , age below 18 years and contact with similar patients are risk factors of pandemic influenza through univariate analysis, whereas none of these parameters were demonstrated to have any statistically significant correlation with a diagnosis of seasonal influenza. Table 2 illustrates a comparison of the incidence rate of major clinical manifestations of disease (fever, cough, body aches and diarrhea) between pandemic and seasonal influenza. As indicated by Table 2 , the only significant difference between pandemic and seasonal influenza was the higher prevalence of gastrointestinal symptoms whereas no significant difference was found between the two groups associated with fever, cough and muscular pain.

Among 112 patients with confirmed diagnosis of influenza, 62 cases with pandemic and 32 with seasonal influenza were hospitalized. Of the hospitalized patients with pandemic influenza, twelve cases were admitted to Intensive Care Units (ICUs) and eleven needed mechanical ventilation while two patients died because of the severity of disease. The number of admissions to ICUs for confirmed cases of seasonal flu was seven patients amongst which, five needed assisted ventilation and eventually one patient failed to recover from the disease. As shown in Table 3, no significant difference concerning these variables was found between the two groups.

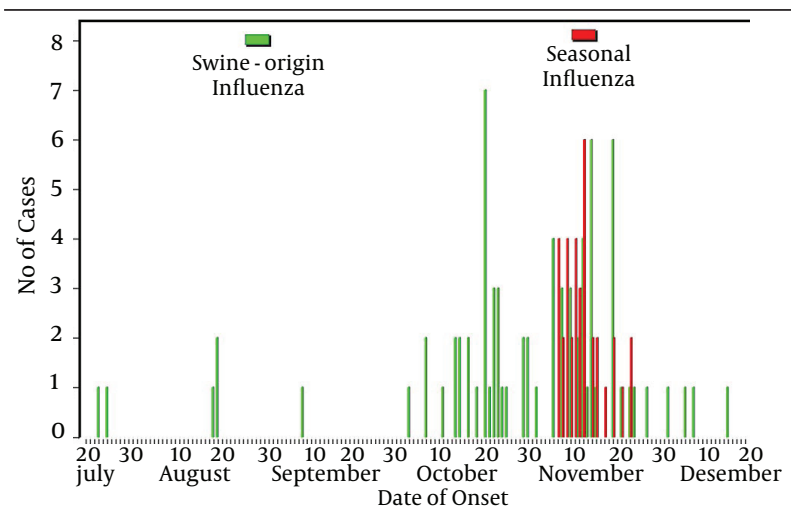

Figure 1. The Epidemiological Curve of Confirmed Cases of Swine-Origin and Seasonal Influenza 
Bijani B et al.

Table 1. Demographic Information of Patients With Swine-Origin and Seasonal Influenza From the Qazvin Province During the 2009 Pandemic ${ }^{a}$

\begin{tabular}{lccc}
\hline Risk factor & Novel Influenza A (H1N1) & Seasonal Influenza & P Value \\
\hline Age $(<\mathbf{1 8})$ & $48 / 76(63.2)$ & $32 / 36(88.9)$ & 0.005 \\
Gender $($ male) & $45 / 76(59.2)$ & $22 / 36(61.1)$ & 0.848 \\
Human contact & $8 / 76(10.5)$ & $1 / 36(2.8)$ & 0.149 \\
International travel & $3 / 76(3.9)$ & $0 / 36(0)$ & 0.308 \\
\hline
\end{tabular}

a No/total (\%).

Table 2. Clinical Manifestations of Swine-Origin and Seasonal Influenza a

\begin{tabular}{lccc}
\hline Clinical Manifestations & Novel Influenza A (H1N1) & Seasonal Influenza & PValue \\
\hline Fever & $70 / 76(92.1)$ & $33 / 36(91.7)$ & 0.599 \\
Cough & $73 / 76(96.0)$ & $33 / 36(91.7)$ & 0.293 \\
Myalgia & $44 / 76(57.9)$ & $14 / 36(38.9)$ & 0.060 \\
Gastro intestinal manifestations & $26 / 76(34.2)$ & $2 / 36(5.5)$ & 0.001 \\
\hline
\end{tabular}

${ }^{\mathrm{a}}$ No/total (\%)

Table 3. Course and Outcome of Swine-Origin and Seasonal Influenza ${ }^{a}$

\begin{tabular}{lccc}
\hline Outcome Variables & Novel Influenza A $\mathbf{( H 1 N 1 )}$ & Seasonal Influenza & PValue \\
\hline Hospital admission & $62 / 76(81.6)$ & $32 / 36(88.9)$ & 0.325 \\
Admission in ICUs & $12 / 76(15.8)$ & $7 / 36(19.4)$ & 0.630 \\
Need for assisted ventilation & $11 / 76(14.5)$ & $5 / 36(13.9)$ & 0.934 \\
Mortality & $2 / 76(2.6)$ & $1 / 36(2.8)$ & 0.691 \\
\hline
\end{tabular}

${ }^{\mathrm{a}}$ No/total (\%).

\section{Discussion}

One of the characteristics of the swine-origin influenza A (H1N1) pandemic was its rapid distribution worldwide, so that only after several months following the report of the first cases of the disease in early 2009, in the second half of the year, the most reported global cases of influenza were due to this virus; in Europe and United States this virus was isolated in more than $99 \%$ of influenza cases (5). In the present study, most confirmed cases of influenza were also associated with swine-origin H1N1 virus; prior to the beginning of the influenza season (until autumn 2009 ), all confirmed cases of influenza and $68 \%$ of cases during the entire course of the study were due to the swine-origin influenza A (H1N1) virus. Another noteworthy epidemiological feature of pandemic influenza was the younger age of the patients. As it is evident, in the present study, the age of patients with swine-origin influenza A(H1N1) was significantly lower than those with seasonal flu. In studies carried out elsewhere, similar results have been reported; in some studies published at the beginning of the epidemic in developed countries, 60\% of patients were 18 years old or younger (2). To elucidate this phenomenon, in addition to prior immunity in older individuals, the higher rate of sampling in the age group younger than 18 years (due to appearance of epidemic in schools) is propounded (6). Considering the antigenic similarities between swine-origin influenza A (H1N1) virus and viruses associated with influenza pandemics over the distant years, a group of American researchers attempted to perform a serological investigation for detection of cross-reactive antibodies against swine-origin H1N1 virus on stored serum samples from many years ago. The results of this study showed that there was a clear difference in antibody titers between the sera of people born before 1950 and those after 1950; the titer of these antibodies was 34\% in people born prior to 1950 and 4\% in those after 1980 (7). As shown in Figure 1, the epidemiological curve is indicative of a person-to-person transmission during the epidemic. The major routes for transmission were contact with fomites contaminated with respiratory or gastrointestinal secretions and through droplets produced during talking, sneezing and coughing $(8,9)$. As evident by the epidemiological curve, the highest number of seasonal influenza occurred during November to December yet pandemic influenza took place in two waves. The first wave appeared from July to October and was mostly found in patients who had returned from Saudi Arabia whereas the second wave emerged after commencement of schools in September. 
In the current study no history of traveling abroad in patients with seasonal flu was observed whereas three cases with novel influenza A (H1N1) had a history of travel to Saudi Arabia and all were infected during July to August 2009. Saudi Arabia had reported the highest number of cases of swine-origin influenza A (H1N1) in the Eastern Mediterranean region until 2009 (10). Furthermore, while investigating the imported cases of disease in Iran until November 2009, the highest number was associated with the pilgrims who had come back from the eastern Mediterranean region. Babak et al report that $52.2 \%$ of imported cases from the Isfahan province were travelers returning from Saudi Arabia (11). Although the symptoms of swine-origin influenza A (H1N1) were to a great extent similar to those of seasonal flu in the recent years, (12) gastrointestinal manifestations, were significantly more prevalent in pandemic influenza in the present study. This is a new feature in flu syndromes, as only $13.4 \%$ of medical students in a cross-sectional research in Karachi, Pakistan reported nausea and vomiting as a symptom of swine-origin influenza A (H1N1) (13). Moreover, as reported by other studies, a higher incidence of myalgia (and even cases of rhabdomyolysis) among patients with swine-origin influenza (14) was also observed in the present study yet this difference was not statistically significant. In other studies carried out in Iran, higher prevalence of myalgia and diarrhea was reported as well. Saleh et al. reported as high as $80 \%$ prevalence for myalgia and $77.5 \%$ for diarrhea in 40 patients from the north west of Iran (15). In spite of initial reports of the high mortality rate in swine-origin influenza (38\% of hospitalized patients in Mexico) (16), the later studies reported a mortality rate for swine-origin influenza comparable with figures found for seasonal influenza. For instance, in a study by Louie et al. a mortality rate of $11 \%$ in hospitalized patients was reported (17). Even in the study of Cao et al. on 426 confirmed cases of swine-origin influenza A (H1N1), admitted to 20 hospitals of China, no mortality was reported (18). Also in another study by Chang et al. from Australia, there was no significant difference in admission to intensive care units, need for assisted ventilation, and mortality rate between patients with swine-origin influenza and those with seasonal flu (19). As mentioned in the results section, no difference in admission to intensive care units, need for assisted ventilation and mortality rate was found between patients with swine-origin influenza A (H1N1) infection and those with seasonal flu. Comparison of swine-origin and seasonal influenza has not been studied in the Eastern Mediterranean region yet the mortality rate for swine-origin influenza has been reported for some territories. A mortality rate of $16.7 \%$ in Isfahan, (20) 20\% in eastern Azerbaijan (15) and 15\% for the pediatric age group in Zahedan (21) has reported. Torun et al. reported $14 \%$ intubation rate in 114 suspected swineorigin influenza A (H1N1) patients in Turkey, who were amongst the pediatric age group. Fortunately, all patients survived in that study (22).
There is only one study comparing swine-origin influenza A (H1N1) with other upper respiratory infection cases (not necessarily seasonal influenza) in Iran, which was conducted in the Hamadan province. In that study, mortality in the former group was $3 \%$, yet none of the patients in later group died. In that study other outcome variables (admission to intensive care units or need to assisted ventilation) were not considered and no significant statistical difference in clinical presentation was found between swine-origin influenza A (H1N1) and other upper respiratory tract infections (23). Considering the occurrence of swine-origin H1N1 infection in younger age groups, the appearance of cases of disease outside the epidemic season, and the association of gastrointestinal symptoms with swine-origin H1N1 infection compared to seasonal flu, accurate attention to these criteria could boost the clinical suspicion of cases of novel influenza in the absence of sufficient diagnostic laboratory facilities.

\subsection{Limitations}

This study was limited to the Qazvin province, situated in the North-West of Iran, in the Eastern Mediterranean region. The relatively low number of cases did not permit us to analyze fatalities in specific populations such as pregnant women and overweigh patients. Performing similar research in different regions of the Middle East could produce more useful information related to differences in underlying factors, clinical manifestations, course of disease and prognosis.

\section{Acknowledgements}

We would like to express our gratitude for all the assistance provided throughout the study by the health care personnel of Qazvin University of Medical Sciences.

\section{Authors' Contributions}

Study concept and design: Behzad Bijani, Reza QasemiBarqi and Mohammad Reza Sarokhani. Acquisition of data: Mohammad Reza Sarokhani, Behzad Bijani and Reza Qasemi-Barqi. Analysis and interpretation of data: Behzad Bijani and Mohammad Reza Sarokhani. Statistical analysis: Behzad Bijani. Drafting of the manuscript: Ali Asghar Pahlevan, Behzad Bijani and Reza Qasemi-Barqi.

\section{References}

1. Dushoff J, Plotkin JB, Viboud C, Earn DJ, Simonsen L. Mortality due to influenza in the United States--an annualized regression approach using multiple-cause mortality data. Am J Epidemiol. 2006;163(2):181-7.

2. Novel Swine-Origin Influenza AIT, Dawood FS, Jain S, Finelli L, Shaw MW, Lindstrom S, et al. Emergence of a novel swineorigin influenza A (H1N1) virus in humans. $N$ Engl $\mathrm{J} \mathrm{Med}$. 2009;360(25):2605-15.

3. Gooya MM, Soroush M, Mokhtari-Azad T, Haghdoost AA, Hemati P, Moghadami M, et al. Influenza A (H1N1) pandemic in Iran: report of first confirmed cases from June to November 2009. Arch Iran Med. 2010;13(2):91-8.

4. World Health Organization. CDC protocol of realtime RT-PCR 
for swine influenza A (H1N1). In: World Health Organization., editor. Geneva: 2009.

5. World Health Organization. Pandemic (H1N1) 2009. In: World Health Organization, editor. Geneva: 2009.

6. Centers for Disease C, Prevention.. Swine-origin influenza A (H1N1) virus infections in a school - New York City, April 2009. MMWR Morb Mortal Wkly Rep. 2009;58(17):470-2.

7. Hancock K, Veguilla V, Lu X, Zhong W, Butler EN, Sun H, et al. Cross-reactive antibody responses to the 2009 pandemic H1N1 influenza virus. NEngl J Med. 2009;361(20):1945-52.

8. Boone SA, Gerba CP. The occurrence of influenza A virus on household and day care center fomites.J Infect. 2005;51(2):103-9.

9. Bean B, Moore BM, Sterner B, Peterson LR, Gerding DN, Balfour HJ. Survival of influenza viruses on environmental surfaces.J Infect Dis. 1982;146(1):47-51.

10. Haworth E, Rashid H, Booy R. Prevention of pandemic influenza after mass gatherings - learning from Hajj. J R Soc Med. 2010;103(3):79-80.

11. Babak A, Akhtar E, Nobari RF, Ataei B. Surveillance on the 2009 pandemic influenza A (H1N1) virus, Isfahan, Iran. J Res Med Sci. 2011;16(1):119.

12. Myers KP, Olsen CW, Gray GC. Cases of swine influenza in humans: a review of the literature. Clin Infect Dis. 2007;44(8):1084-8.

13. Khowaja ZA, Soomro MI, Pirzada AK, Yoosuf MA, Kumar V. Awareness of the Pandemic H1N1 Influenza global outbreak 2009 among medical students in Karachi, Pakistan.J Infect Dev Ctries. 2011;5(3):151-5.

14. Ayala E, Kagawa FT, Wehner JH, Tam J, Upadhyay D. Rhabdomyolysis associated with 2009 influenza A(H1N1). JAMA. 2009; 302(17):1863-4.

15. Saleh PNH, Naghili B. Clinical manifestations of patients with novel H1N1 infection hospitalized in Infectious Disease ward,
Sina hospital, Tabriz, Iran. Iran J of Clinical Infectious Diseases. 2010;5(4):200-5.

16. Perez-Padilla R, de la Rosa-Zamboni D, Ponce de Leon S, Hernandez M, Quinones-Falconi F, Bautista E, et al. Pneumonia and respiratory failure from swine-origin influenza A(H1N1) in Mexico. N EnglJ Med. 2009;361(7):680-9.

17. Louie JK, Acosta M, Winter K, Jean C, Gavali S, Schechter R, et al. Factors associated with death or hospitalization due to pandemic 2009 influenza $\mathrm{A}(\mathrm{H} 1 \mathrm{~N} 1)$ infection in California. JAMA. 2009;302(17):1896-902.

18. Cao B, Li XW, Mao Y, Wang J, Lu HZ, Chen YS, et al. Clinical features of the initial cases of 2009 pandemic influenza A(H1N1) virus infection in China. N EnglJ Med. 2009;361(26):2507-17.

19. Chang YS, van Hal SJ, Spencer PM, Gosbell IB, Collett PW. Comparison of adult patients hospitalised with pandemic (H1N1) 2009 influenza and seasonal influenza during the "PROTECT" phase of the pandemic response. Med J Aust. 2010;192(2):90-3.

20. Javadi AA, Ataei B, Khorvash F, Babak A, Rostami M, Mostafavizadeh K, et al. Clinical features of novel 2009 influenza A (H1N1) infection in Isfahan, Iran.J Res Med Sci. 2011;16(12):1550-4.

21. Soleimani G, Akbarpour M. Clinical presentation of novel influenza a $(\mathrm{h}(1) \mathrm{n}(1))$ in hospitalized children. Iran J Pediatr. 2011;21(2):215-9.

22. Torun SH, Somer A, Salman N, Ciblak M, Demirkol D, Kanturvardar M, et al. Clinical and epidemiological characteristics of pandemic influenza $\mathrm{A} /(\mathrm{H} 1 \mathrm{N1})$ in hospitalized pediatric patients at a university hospital, Istanbul, Turkey. J Trop Pediatr. 2011;57(3):213-6.

23. Cheraghi Z, Doosti Irani A, Rezaiean S, Ahmadzadeh J, Poorolajal J, Erfani H, et al. Influenza A (H1N1) in Hamedan Province, Western Iran in 2009: A Case-Control Study. J Res Health Sci. 2010;10(1):15-21. 